\title{
LESSONS LEARNED IN THE DEVELOPMENT OF AN ONLINE 6-3-5 DIGITAL DESIGN TOOL FOR DISTRIBUTED IDEA GENERATION
}

\author{
Ross BRISCO, Hilary GRIERSON and Andrew LYNN \\ Department of Design, Manufacturing and Engineering Management, University of Strathclyde, \\ United Kingdom
}

\begin{abstract}
In a year, which has observed a change towards more online teaching and learning, there is still a limited number of online collaborative design tools which can support distributed design activities. A prototype online tool to support 6-3-5 (known as the 6-3-5 method or brainwriting) was developed, at the Department of Design, Manufacturing and Engineering Management (DMEM), University of Strathclyde, to support idea generation. The software is accessed through a web browser and guides through the 6-3-5 method. Students can conduct the activity in a synchronous mode (live) supported by text or video chat, or in an asynchronous mode. This paper discusses the design and development of the online 6-3-5 tool and key requirements for its further development including its suitability for both use by distributed design teams and as an educational tool. The outcomes of this research are the lessons learnt in developing the prototype online tool, its evaluation and the identification of further creative methods that could be digitised in the future.
\end{abstract}

\section{Keywords: Distributed design, design activity, creative design methods, 6-3-5 method, design tools}

\section{INTRODUCTION}

Design methods allow for the creation of design ideas using recognised processes. Design methods are well established, used in industry and education to achieve a particular goal to meet outcomes. While collaboration and communication tools have developed with new computer technologies, there appears to be fewer digital/online creative design methods compared to physical creative methods.

Digital design methods support design activities and involve using computer devices, allowing for design ideation to occur. This can be collocated or distributed. One of the most common online tools is the digital whiteboard, where many users can contribute ideas as reported in papers such as Vidovics et al [1]. Design activities conducted on a digital whiteboard tend to replicate those on a physical whiteboard in a collocated space. This realisation can be compared to many of the digital design online tools using existing web 2.0 functionality. They manage and capture the design process, e.g., storing design documentation in a prescribed way or recoding and digitising design outputs. Several developments on a shared whiteboard have emerged and are commonly used by design teams including Miro (miro.com), Mural (mural.co), Sketchboard (sketchboard.io) and others. Some include specific templates for designers including decision making frameworks or ideation brainwriting.

There are many case studies reported in the literature of distributed design teams conducting design activities as online technology capabilities grow, including synchronous CAD [2], social network sites [3], collaborative document editors [4], learning management systems [5], and video conference [6] for a range of design activities including design review meetings, democratic decision making (voting/polling), annotating concepts, CAD development and a range of design methods including TRIZ, design alternatives, market research and feedback from stakeholders.

Over time, there have been novel developments of design methods and tools which are inspired by computer functionality. ICR (Inform, Create, Reflect) is a digital design activity using Microsoft OneNote, which enables design teams to collaborate on the development of ideas by passing and adding information to concepts [7]. The ICR method supports more complex and developed ideas compared to other design methods such as 6-3-5. However, the novelty of the concepts is questionable and significantly less concepts are generated by team members than with physical 6-3-5. Sketchy [8] is a 
digital design tool that allows designers to draw ideas on their own mobile or computer device individually, but they can be inspired by other collaborators using a 'peek' feature. 'Peek' allows a design team member to quickly watch another team member who is also drawing in real-time. The designer then can update their own ideas based on inspiration from others.

\subsection{Experiences of the 6-3-5 Method}

6-3-5 is a design activity similar to brainstorming but with more benefits, including extended thinking time for participants and reduced impact of dominant team members [9]. The 6-3-5 method has been attributed to encouraging more creative concepts, especially for students who have skills in longer concept generation projects, such as mechanical engineering students [10]. For educators, 6-3-5 is simple enough to explain, conduct, discuss and reflect upon during a typical tutorial session [11] which perhaps explains its popularity within an educational setting. Furthermore, students are more likely to apply design methods in the future when they learn by experiencing the technique within exercises or workshops [11].

At DMEM, University of Strathclyde, students utilise computer technology to support distributed collaboration when physical collaboration is impossible. The design method which is most commonly and successfully used to generate ideas, face-to-face, has been the 6-3-5 design method, where six team members are challenged to draw three ideas in five minutes. After the five minutes, the team members swap drawings, develop the other team members ideas for another five minutes, and so on for six rounds. In a distributed environment, students typically facilitate this design method by drawing their own three ideas and then photographing or scanning the ideas and sending them to their fellow team members. This is complex and time-consuming; however, students appreciate the systematic nature of this design process.

Due to successful use of the 6-3-5 tool within distributed collaboration projects, there was a motivation to investigate if an online version of 6-3-5 could be developed that supports online idea generation and also educators in teaching the method. In this paper, the requirements of an online 6-3-5 tool are described, the development of a prototype online 6-3-5 tool is documented and an evaluation of its use in a student design class is reported. This paper shares the considerations of building the online 6-3-5 design tool, the process undertaken to develop the tool and the lessons learnt which may influence others who are considering developing online creative design tools.

\section{BUILDING AN ONLINE DISTRIBUTED TOOL TO SUPPORT 6-3-5}

The online 6-3-5 tool was inspired by the game Drawful TM (jackboxgames.com/drawful) and subsequent versions of the game by developer Jackbox Games. In this game, players draw an image on a mobile device based on a prompt. All players then vote on the 'best' image, which meets the prompt. This has analogies with creative design methods where designers generate ideas based on a brief, by drawing concepts and then selecting the most suitable idea based on user requirements. As Drawful ${ }^{\mathrm{TM}}$ is a party game that is often played with friends, it was considered that a similar tool could be developed which supports the creative design process. A design method was required that enabled individuals to generate many ideas by drawing. This is where the success of 6-3-5 within DMEM and the implementation of a drawing design method came together. The success of the 6-3-5 in classes to produce numerous ideas and the playfulness of the online party game were the basis of the requirements of the 6-3-5 online tool.

\subsection{Requirements of the 6-3-5 tool}

Requirements for a digital creative design tool cover a range of categories, including functional, data, environmental and contextual, user-centred and usability [7]. The authors used this work to establish the requirements of the 6-3-5 online tool (see table 1).

The requirements identified a need for the host of the instance (in other words a 6.3 .5 session) to alter the rules of 6-3-5 based upon the number of participants. Also, there is a need for the participant to be guided through the 6-3-5 method if they are new to the tool. This led to the realisation that this digital tool could be a useful educational resource for students and others who are new to the 6-3-5 method of generating ideas. The requirements of this tool were established as: 
1. Based upon the established requirements for an online 6-3-5 tool, a user journey was created to communicate the sequence of events to the programmer to set up and conduct a digital 6-3-5 activity. This included five critical areas of the digital tool: A 'Start' page where a host could setup the instance or a participant can join.

2. A 'New instance' page featured a setting for the number of participants, time per round and number of rounds. The User can enter the 'theme' of the drawing ideas at this time.

3. A 'Joining information' page where the User can join a digital 6-3-5 instance.

4. A 'Drawing' page where the User draws their ideas, with subsequent pages for each round.

5. A 'View and download/ screen for all ideas generated.

Pages from the visual user journey document are illustrated in Figure 1.

Table 1. Requirements for an online 6-3-5 tool

\begin{tabular}{|l|l|}
\hline Functional & $\begin{array}{l}\text { The User should be limited by the rules of 6-3-5. } \\
\text { The host should be able to change the rules-based upon the } \\
\text { requirements of the task (e.g., more time, less participants etc). }\end{array}$ \\
\hline Data & $\begin{array}{l}\text { The User and the host can view all drawings digitally and } \\
\text { download each drawing. }\end{array}$ \\
\hline Environmental and contextual & $\begin{array}{l}\text { The system should be easy to access on a computer device. } \\
\text { The system should allow drawing with a mouse or touchscreen. }\end{array}$ \\
\hline User-centred & $\begin{array}{l}\text { Instructions should be easy to follow, including for those who } \\
\text { have never conducted a 6-3-5 before. }\end{array}$ \\
\hline Usability & The User should be guided through the 6-3-5 process. \\
\hline
\end{tabular}

From discussions with the programmer, a methodology was agreed for the creation of the online tool. Due to the complexity of developing a web app and mobile app, the programmer suggested working on a prototype web app first, to ensure a working prototype.
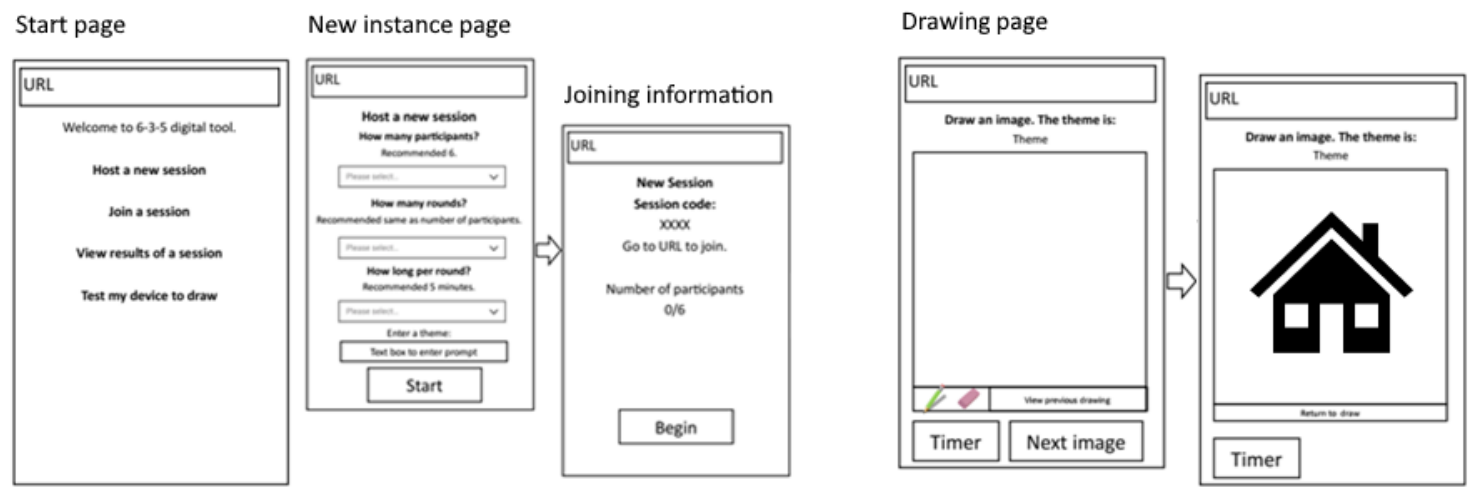

Figure 1. Example pages from the visual user journey document

\subsection{Coding the prototype 6-3-5 online tool and its functionality}

The 6-3-5 online software was written in PHP, Smarty and JavaScript, and stores its data in a MySQL database. The software allows a number of participants to be specified for the activity from a pool of registered users by the instance owner. When an owner starts an instance, the database is populated with a unique identifier for the input drawing for each User, for each round, of the entire 6-3-5 instance - this is achieved by an algorithm that iterates over arrays of users, and their position in each round. This algorithm can accept a variable number of users, as required for each instance.

As users complete drawing tasks and submit them back to the system (as base64-encoded image data along with encoded identifiers and metadata), it records the completion of an individual drawing and then creates links for users in their 'to-do' list of drawing tasks. Users can only begin their drawings when the prerequisite input drawing is available. The required drawing functionality for the 6-3-5 task is generated via a drawing module modified from an open-source painting package named miniPaint (available at: github.com/viliusle/miniPaint).

Depending on the round and number of participants, at any given instant, each participant can have different amounts of drawing tasks available for them to perform, and those drawing tasks can be from 
several different rounds of the overall instance. A user can participate in any number of 6-3-5 instances concurrently. Owners of a 6-3-5 instance can see all drawings from all participants at any time. Participants can see all drawings in all 6-3-5 instances they have participated in only when that instance has concluded, or the owner has ended it.

\section{USING THE 6-3-5 ONLINE TOOL WITHIN AN EDUCATIONAL SETTING}

With a prototype created, the next step was to evaluate the prototype 6-3-5 online tool in its initial form. To support the evaluation, a framework was developed with university students, some who had used 63-5 before and others who were learning to use 6-3-5 for the first time.

\subsection{Research Methodology}

The prototype 6-3-5 online tool was introduced to third year students of the DM306 Product Development class at DMEM. There are 95 students in this class, working in teams of 5. Students had two-year's experience with design methods and were new to the 6-3-5 method. Students were introduced to the 6-3-5 paper-based method and then introduced to the prototype 6-3-5 online tool. The class was hosted on Zoom (due to Coronavirus) and utilised breakout rooms to allow each team to complete the 6-3-5 activity using the prototype 6-3-5 online tool. The prompt for the 6-3-5 activity differed across each team, based on the established problem areas they were investigating. Teams were supported by two class lecturers, two class tutors and a facilitator who led the workshop. Staff switched between breakout rooms to ensure students could use the prototype 6-3-5 online tool and understood the 6-3-5 method as they progressed. A survey was created to capture the students' opinions. It queried the prototype 6-3-5 online tool and comparisons with paper based 6-3-5 and requested suggestions for improvement.

\subsection{Survey Results}

29 students responded to the survey, two of whom had experience with the 6-3-5 method before.

One of the aims of the 6-3-5 online tool was to guide students through the 6-3-5 method to educate them on how the method works. Students were asked if, in their opinion, the prototype 6-3-5 online tool guided them through the method. 15 Students said, 'yes, they were guided' and 14 students said, 'no, they were not guided'.

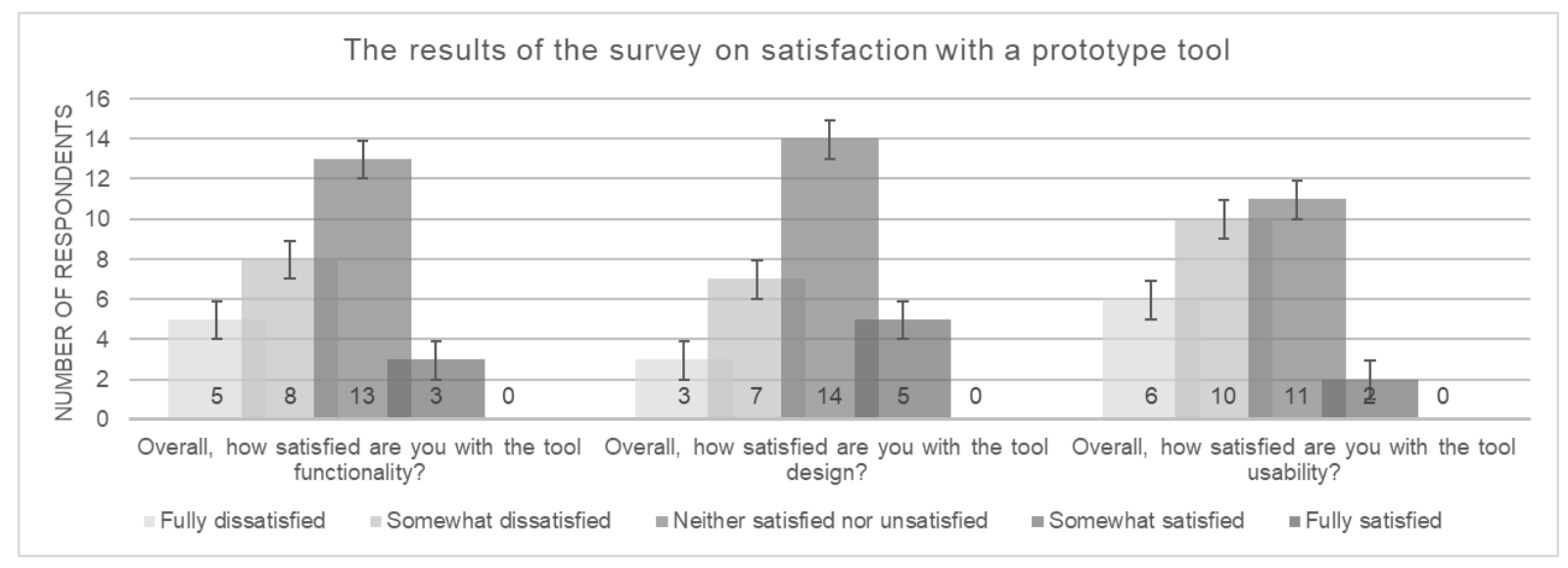

Figure 2. Results of a survey on satisfaction of the functionality, design and usability

On a scale of 1-5 from not satisfied to fully satisfied, students were asked three questions: (1) Overall, how satisfied are you with the 6-3-5 online tool functionality? (2) Overall, how satisfied are you with the 6-3-5 tool design? (3) Overall, how satisfied are you with the 6-3-5 online tool usability? Responses can be seen in Figure 2 including error bars of the sample of respondents (29) against the population of the class (95) with standard deviation of \pm 0.911 . For each of the questions, the most popular response was 3 (neither satisfied nor unsatisfied) - 13 responses for functionality, 14 for design and 11 for usability. Responses were slightly more positive towards satisfaction for design and less favourable for usability. The two students who had experienced 6-3-5 before preferred the paper-based 6-3-5 method and noted that they would use paper based in the future. 


\section{DISCUSSION}

The development of the prototype 6-3-5 online tool came out of a necessity to move to working/studying online. It was difficult to conduct a 6-3-5 creative activity online during class, and so a tool that guided the students through the 6-3-5 process was developed. Based upon the response from students, there were some issues with the prototype 6-3-5 online tool. Only $51 \%$ of the students said that the prototype 6-3-5 online tool guided them through the 6-3-5 process. Student feedback suggested some functionality improvements, such as more informative instructions

The students did not 'learn by doing' whilst using the new online tool. When conducting 6-3-5 on paper, students needed to fully understand the process. Across Zoom, explanation was confusing for students as there was no practical activity to support understanding. When conducting the 6-3-5 method using the prototype 6-3-5 online tool, the automated mode diminished their understanding. This should be cautioned against within an educational environment.

The students found issues which may have influenced their scores, including team members names being missed and overcomplexity of the drawing tool. These were not expected to influence opinions of the prototype 6-3-5 online tool as much as they did. This proved to be good initial feedback.

However, these were not the main issues with the response to the satisfaction questions. In terms of functionality, students considered the prototype 6-3-5 online tool satisfactory. Students noted that functionally was complex. For example, the drawing module offered too many drawing options. The drawing module was as complex as a photoshop clone when all that was required was a pen tool and an eraser tool. The drawing module responded in ways that the students were not expecting. For example, students anticipated drawings would be saved automatically; the default colour was green rather than black, and it was not easy to delete or erase parts of the drawing. These aspects influenced the student's evaluation of the prototype 6-3-5 online tool but can be fixed easily with a simpler drawing module. Usability scored lower for similar reasons.

Another concern based on the survey results was the fact two students who used 6-3-5 before preferred a paper-based 6-3-5 mode, even if improvements were made. This was based on students' dissatisfaction with digital capabilities and the complexity of a digital tool compared to paper. This was unexpected as the tool had been based on the Drawful ${ }^{\mathrm{TM}}$ game. It could be considered that without enjoyment, the digital tool no longer becomes an attractive alternative to physical drawing. It should be noted that the sample size of two is not significant enough to make meaningful decisions about the development of the prototype 6-3-5 online tool but is an indication to investigate further.

overall, considering the requirements in The requirements identified a need for the host of the instance (in other words a 6.3.5 session) to alter the rules of 6-3-5 based upon the number of participants. Also, there is a need for the participant to be guided through the 6-3-5 method if they are new to the tool. This led to the realisation that this digital tool could be a useful educational resource for students and others who are new to the 6-3-5 method of generating ideas. The requirements of this tool were established as: 6. Based upon the established requirements for an online 6-3-5 tool, a user journey was created to communicate the sequence of events to the programmer to set up and conduct a digital 6-3-5 activity. This included five critical areas of the digital tool: A 'Start' page where a host could setup the instance or a participant can join.

7. A 'New instance' page featured a setting for the number of participants, time per round and number of rounds. The User can enter the 'theme' of the drawing ideas at this time.

8. A 'Joining information' page where the User can join a digital 6-3-5 instance.

9. A 'Drawing' page where the User draws their ideas, with subsequent pages for each round.

10. A 'View and download/ screen for all ideas generated.

Pages from the visual user journey document are illustrated in Figure 1.

Table 1, the majority of students are satisfied with the prototype 6-3-5 online tool. In terms of Functionality the 6-3-5 rules have been implemented and the host can change the rules. This indicated that the 6-3-5 method can be transferred to a digital platform. In relation to Data and the User, the host of an instance can view and download all drawing ideas. Regarding Environmental and Contextual issues, the system was easy to access through a URL, and allowed for drawing with a mouse. Other drawing methods such as touchscreen of digital pen should be investigated. To improve user-centred design aspects, the instructions will be made easier to follow on-screen with a more intuitive process. Finally, usability requires some significant changes to allow the participants to conduct a 6-3-5 online and to be guided through the process more readily. 


\subsection{Future development of the 6-3-5 online tool}

The prototype was a significant first step as a proof of concept, but highlighted areas critical to the development which were not considered within the initial requirements. The first change is to simplify the introduction of the 6-3-5 online tool and the instructions on how to use the system. Information on what to do should be delivered as it is required. This will improve its use as an educational tool. The second change is to simplify the 6-3-5 online tool to remove unnecessary functionality. This change will simplify the drawing experience to allow quick ideas to be generated. The third change is to make the 6-3-5 online tool more enjoyable to use. Indeed, the change of input. from mouse to digital pen or touchscreen would be a first step. It is reported that mood and visual stimulus can affect the creativity of outcomes from a 6-3-5 activity [8], and the suggested changes would encourage more creative outcomes.

The low sample size of the students who had used 6-3-5 before impacted the results. A further study with students experienced in 6-3-5 will reveal accurate results supporting the next version of the 6-3-5 online tool. There is also a need to conduct further studies with those unfamiliar with 6-3-5 to determine the level of simplicity required to help with student understanding of the method.

\section{CONCLUSIONS}

This paper documents the early development and evaluation of a prototype 6-3-5 online tool to support distributed idea generation. The reporting of this tool's development and data on how students responded to its use is novel. The need for a 6-3-5 online tool was established from observations of a distributed design class where the 6-3-5 method of producing concepts was successful in supporting global design. The inspiration for the 6-3-5 online tool came from a party videogame that influenced the tool's design. These factors generated requirements for the 6-3-5 online tool and created a user journey map. The web app was coded to the rules of the 6-3-5 method and the requirement to allow multiple instances at once. To evaluate the tool, students were asked to use it within distributed design teams. The results highlighted several areas for improvement and identified educational aspects such as understanding of the 6-3-5 method and the comparison of the digital tool to pen and paper. Aspects in the development of the 6-3-5 online tool, which were expected to be entertaining, conversely added complexity to the method and require simplifying and increasing engagement. The next development of the 6-3-5 online tool will be undertaken in the 2021-22 DMEM classes.

\section{REFERENCES}

[1] Vidovics B., Vukasinovic N., Pavkovic N. and Kovacevic A. "Development of methodology for distributed collaborative design environment," in DS 83: Proceedings of the 18th International Conference on Engineering and Product Design Education (E\&PDE16), 2016.

[2] Phadnis V. S., Leonardo K. A., Wallace D. R. and Olechowski A. L. "An exploratory study comparing CAD tools and working styles for implementing design changes," in Proceedings of the Design Society: International Conference on Engineering Design, 2019, pp. 1383-1392.

[3] Brisco R., Whitfield R. I., Grierson H. et al. "Are social network sites the future of engineering design education?" in DS 93: Proceedings of the 20th International Conference on Engineering and Product Design Education (E\&PDE 2018), Dyson School of Engineering, Imperial College, London. 6th-7th September, 2018, pp. 400-405.

[4] Brisco R., Whitfield R. I., Grierson H., Bohemia E. et al. "Overcoming the challenges of global collaboration through design education," in DS 95: Proceedings of the 21 st International Conference on Engineering and Product Design Education (E\&PDE 2019), University of Strathclyde, Glasgow. 12th-13th September, 2019.

[5] Becattini N., Škec S., Pavković N. and Cascini G. "E-learning infrastructure prototype for geographically distributed project-based learning," in Proceedings of the Design Society: DESIGN Conference, 2020, vol. 1, pp. 1667-1676.

[6] Leung W., Wang Y., Kim S.-W. and et a. "Global Product Development: project-based multidisciplinary joint course," in DS 95: Proceedings of the 21st International Conference on Engineering and Product Design Education (E\&PDE 2019), University of Strathclyde, Glasgow. 12th-13th September 2019.

[7] Wodehouse A. and Ion W. "Augmenting the 6-3-5 method with design information," Research in Engineering Design, vol. 23, no. 1, pp. 5-15, 2012. 
[8] Wallace S., Le B., Leiva L. A., Haq A., Kintisch A., Bufrem G., Chang L. and Huang J. "Sketchy: Drawing Inspiration from the Crowd," Proceedings of the ACM on Human-Computer Interaction, vol. 4, no. CSCW2, pp. 1-27, 2020.

[9] Litcanu M., Prostean O., Oros C. and Mnerie A. V. "Brain-writing vs. Brainstorming case study for power engineering education," Procedia-Social and Behavioral Sciences, vol. 191, pp. 387390, 2015.

[10] De Napoli L., Rizzuti S.et al. "Product design education in mechanical and management engineering master's degree programs. Analogies and differences," in DS 104: Proceedings of the 22nd International Conference on Engineering and Product Design Education (E\&PDE 2020), VIA Design, VIA University in Herning, Denmark. 10th-11th September, 2020.

[11] Nutzmann M., Sauer T., VOß M., Bozkurt H. et al. "Study on learning concepts applying creativity methods in education and industry," in DS 95: Proceedings of the 21st International Conference on Engineering and Product Design Education (E\&PDE 2019), University of Strathclyde, Glasgow. 12th-13th September, 2019. 